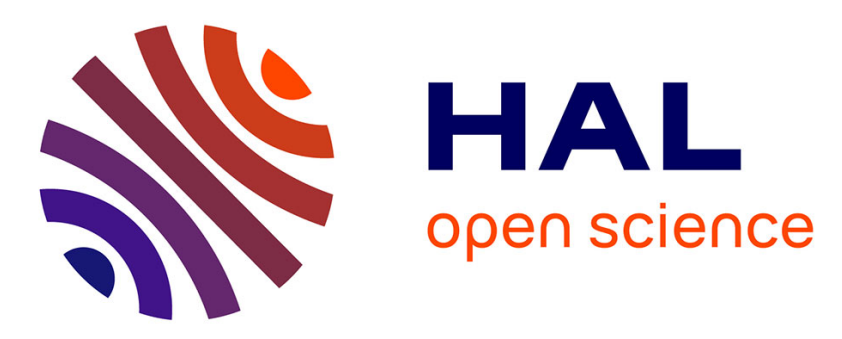

\title{
Étude mathématique de la réponse optique d'une structure nématique en hélice distordue par un champ normal à son axe
}

F. Gharadjedaghi, J. Robert

\section{- To cite this version:}

F. Gharadjedaghi, J. Robert. Étude mathématique de la réponse optique d'une structure nématique en hélice distordue par un champ normal à son axe. Revue de Physique Appliquée, 1975, 10 (5), pp.281-283. 10.1051/rphysap:01975001005028100 . jpa-00243913

\section{HAL Id: jpa-00243913 https://hal.science/jpa-00243913}

Submitted on 1 Jan 1975

HAL is a multi-disciplinary open access archive for the deposit and dissemination of scientific research documents, whether they are published or not. The documents may come from teaching and research institutions in France or abroad, or from public or private research centers.
L'archive ouverte pluridisciplinaire HAL, est destinée au dépôt et à la diffusion de documents scientifiques de niveau recherche, publiés ou non, émanant des établissements d'enseignement et de recherche français ou étrangers, des laboratoires publics ou privés. 


\author{
Classification \\ Physics Abstracts \\ $2.420-7.130-8.816$
}

\title{
ÉTUDE MATHÉMATIQUE DE LA RÉPONSE OPTIQUE D'UNE STRUCTURE NÉMATIQUE EN HÉLICE DISTORDUE PAR UN CHAMP NORMAL A SON AXE
}

\author{
F. GHARADJEDAGHI et J. ROBERT
}

C. E. A.-C. E. N. G., Laboratoire Electronique et de Technologie de l'Informatique Laboratoire de Microélectronique Physique, B. P. 85, Centre de tri, 38041 Grenoble Cedex, France

(Reçu le 26 février 1975, accepté le 25 avril 1975)

\begin{abstract}
Résumé. - Nous avons étudié la distorsion d'une structure nématique en hélice par un champ électrique perpendiculaire à l'axe de l'hélice. Les comportements statiques et dynamiques des effets électro-optiques correspondants sont analysés mathématiquement.
\end{abstract}

\begin{abstract}
The distortion of a twisted nematic structure induced by an electric field normal to the helical axis was studied. The connecting electro-optical static and dynamic behaviours are mathematically analysed.
\end{abstract}

Dans une structure nématique en hélice un champ électrique (ou magnétique) normal à l'axe de l'hélice induit une distorsion [1], [2], [3], qui crée un effet optique. Nous considérons un milieu limité suivant l'axe de l'hélice, et illimité suivant la direction du champ (Fig. 1).

\section{$\otimes \quad$ analyseur $(\perp \vec{E})$}

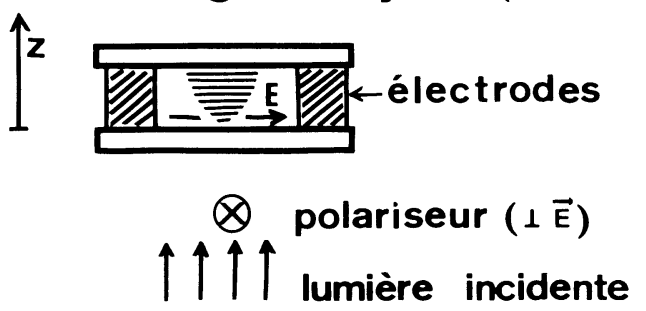

Fig. 1. - Schéma de la cellule.

Le champ électrique est homogène en tous points de la cellule puisque sa composante suivant l'axe de l'hélice et son rotationnel sont nuls.

Nous avons calculé numériquement la distorsion de la structure ainsi que la réponse optique de l'effet induit, entre polariseur et analyseur parallèles; l'expression mathématique régissant la distorsion statique de la structure, déduite de la minimisation de l'équation de l'énergie du milieu est donnée par [3] :

$$
z=\frac{1}{E} \sqrt{\frac{K_{22}}{\varepsilon_{\mathrm{a}}}} \cdot k \cdot F(k, \varphi) ;
$$

et celle correspondant au régime dynamique par [4] :

$$
K_{22} \frac{\partial^{2} \varphi}{\partial z^{2}}+\frac{\varepsilon_{\mathrm{a}} E^{2}}{8 \pi} \sin 2 \varphi=\gamma_{1} \frac{\partial \varphi}{\partial t}
$$

avec :

$\varphi$ : angle du directeur par rapport à $O x ;$

$K_{22}$ : constante élastique de torsion ;

$\varepsilon_{\mathrm{a}}=\varepsilon_{\|}-\varepsilon_{\perp}$ : anisotropie diélectrique $\mathrm{du}$ cristal liquide (on choisit $\varepsilon_{\mathrm{a}}>0$ ) ;

$k$ : constante d'intégration déterminée par les conditions aux limites ;

$\gamma_{1}$ : un des coefficients de viscosité ;

$F(k, \varphi)$ : intégrale elliptique de $1^{\text {re }}$ espèce.

On démontre que l'évolution de l'onde optique se propageant dans le milieu suivant $O z$ est donné par le système suivant :

$$
\begin{aligned}
& \frac{\partial}{\partial z} F_{1}=j \pi \frac{\Delta n}{\lambda} F_{2} \mathrm{e}^{2 j \varphi} \\
& \frac{\partial}{\partial z} F_{2}=j \pi \frac{\Delta n}{\lambda} F_{1} \mathrm{e}^{-2 j \varphi}
\end{aligned}
$$

avec :

$\Delta n=n_{\mathrm{e}}-n_{0}:$ biréfringence du cristal liquide ;

$\lambda$ : longueur d'onde de la lumière ; avec :

$$
F_{1}=D_{x}+j D_{y} \quad \text { et } \quad F_{2}=D_{x}-j D_{y} .
$$

$D_{x}$ et $D_{y}$ sont les composantes du vecteur d'induction électrique de l'onde optique. 
Nous avons résolu les équations ci-dessus en donnant au paramètre $K_{22} / \gamma_{1}$ la valeur de $3,2 \times 10^{-7} \mathrm{C}$. G. S. et au paramètre $\pi \Delta n / \lambda$ la valeur de $1(\mu \mathrm{m})^{-1}$ et en prenant comme épaisseur de la cellule $L=7,5 \mu \mathrm{m}$. La figure $2 a$ donne la distorsion de la structure en régime statique pour différentes valeurs du rapport $E / E_{0}$ avec

$$
E_{0}=\frac{\pi^{2}}{2 L} \sqrt{\frac{\pi K_{22}}{\varepsilon_{\mathrm{a}}}} .
$$

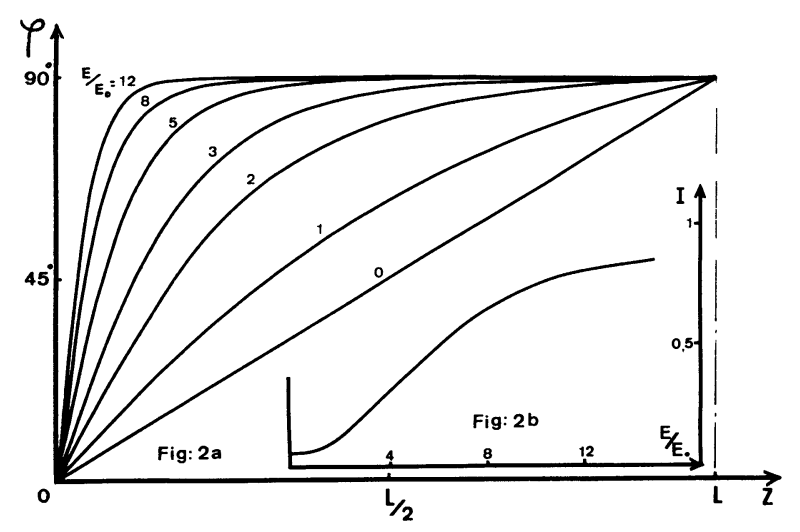

FIG. $2 a$. - Distorsion de la structure en régime statique.

Fig. $2 b$. - Coefficient de transmission en fonction de $E / E_{0}$ en régime statique.

Ce réseau de courbes permet ensuite de calculer, par le système [3], le coefficient de transmission de la lumière en fonction de $E / E_{0}$ (Fig. $2 b$ ).

L'évolution en fonction du temps de la distorsion a été représentée, pour la montée sur la figure 3 et pour la décroissance sur la figure 4 avec $E / E_{0}=8$.

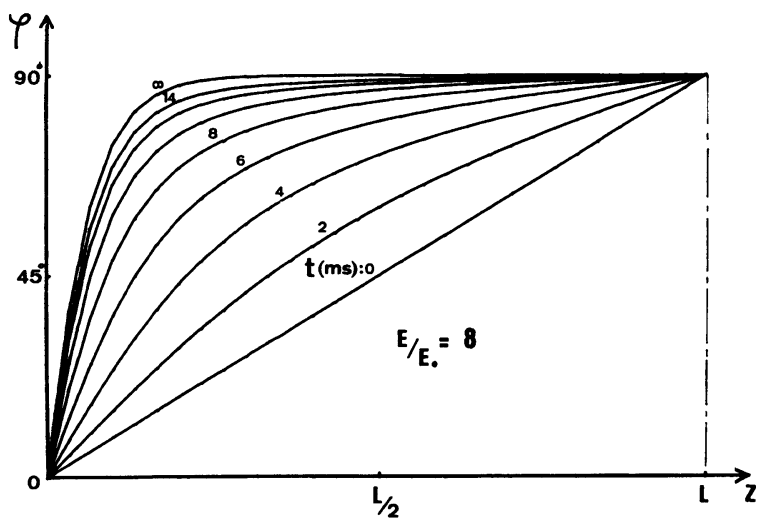

Fig. 3. - Distorsion de la structure en régime dynamique (montée).

La réponse optique, en régime transitoire, correspondant à $E / E_{0}=4,6,8$ est donnée, sur la figure $5 a$. En examinant les figures 3,4 et $5 a$, on constate que l'effet optique n'apparaît qu'après $6 \mathrm{~ms}$ environ, et la structure présente alors une distorsion importante. De même lors de la décroissance, l'effet optique disparaît pratiquement après $20 \mathrm{~ms}$, bien avant que la structure revienne à l'état d'équilibre. En effet, la constante de temps de décroissance de la distorsion de la structure,

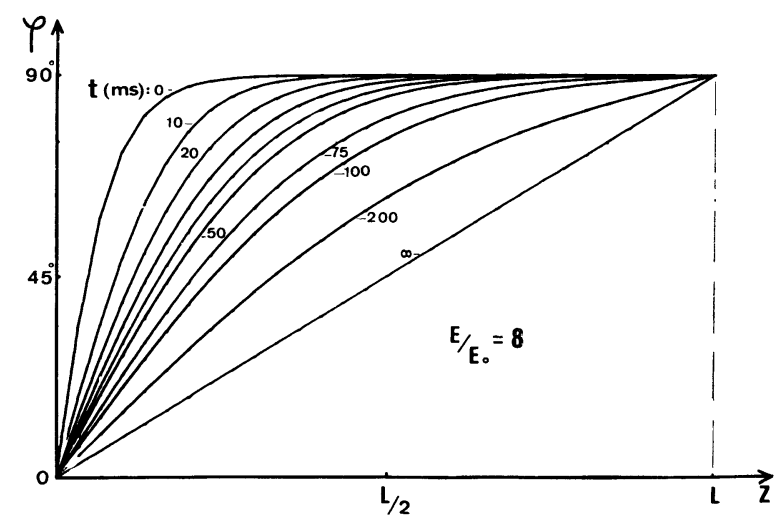

Fig. 4. - Distorsion de la structure en régime dynamique (décroissance).

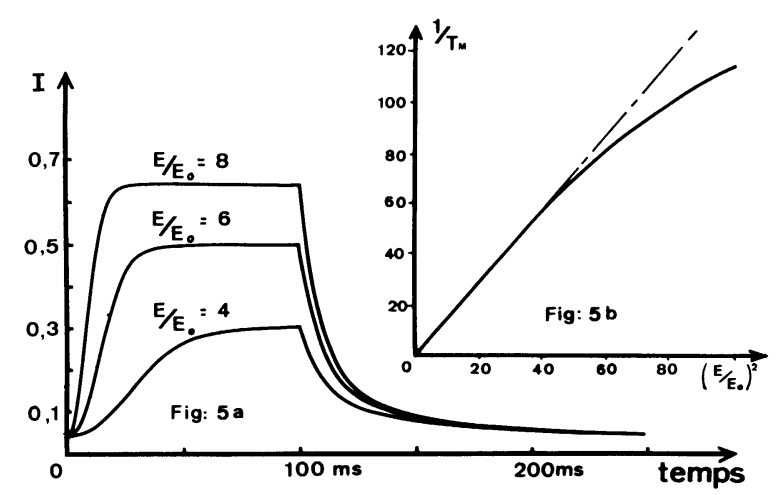

Fig. 5a. - Réponses optiques transitoires (montée et décroissance).

Fig. 5b. - Inverse du temps de montée de l'effet optique en fonction de $\left(E / E_{0}\right)^{2}$.

déterminée à partir de l'éq. (5) (voir plus loin), est de l'ordre de $170 \mathrm{~ms}$; or la constante de temps de décroissance de l'effet optique, qui ne dépend pas de l'excitation antérieure, est ici de l'ordre de $15 \mathrm{~ms}$.

La montée de l'effet optique présente un point d'inflexion et la pente en ce point peut caractériser le temps de montée. La figure $5 b$ représente l'inverse de ce temps de montée en fonction de $\left(E / E_{0}\right)^{2}$. La courbe est linéaire pour des champs faibles $\left(E / E_{0}<6\right)$.

Cas particulier. - Pour de faibles distorsions, la résolution analytique des éq. (1) et (2) est possible. On trouve, en régime statique, la solution :

$$
\varphi=q_{0} z+a_{0} \sin 2 q_{0} z \text { avec } q_{0}=\pi / 2 L
$$

et

$$
a_{0}=\frac{1}{32 \pi q_{0}^{2}} \cdot \frac{\varepsilon_{\mathrm{a}} E^{2}}{K_{22}} .
$$

Pour trouver la réponse du phénomène à un échelon de champ électrique, nous cherchons une solution de la forme $\varphi(z, t)=q_{0} z+a(t) \sin \left(2 q_{0} z\right)$ et $a(t)$ ne prenant que des valeurs faibles.

Les expressions correspondant respectivement au 
phénomène de montée et au phénomène de décroissance s'écrivent :

$a(t)=\frac{B}{m} \cdot \frac{t h m t}{1+\frac{A}{2 m} \cdot t h m t}$ pour la montée

$a(t)=a_{1} \exp \left[-4 q_{0}^{2} \cdot \frac{K_{22}}{\gamma_{1}} \cdot t\right]$ pour la décroissance

avec

$$
A=4 q_{0}^{2} \frac{K_{22}}{\gamma_{1}}, \quad B=\frac{\varepsilon_{\mathrm{a}} E^{2}}{8 \pi \gamma_{1}}
$$

et

$$
m=\sqrt{8 B^{2}+A^{2}}
$$

$a_{1}$ représente l'amplitude de la distorsion induite et est égal à $a_{0}$ dans le cas de l'application d'un échelon de champ de faible amplitude.

En choisissant l'inverse de la pente à l'origine de la fonction $a(t)$ [4] comme constante de temps de montée $T_{\mathrm{m}}$ du phénomène, on obtient :

$$
T_{\mathrm{m}}=\frac{1}{B}=\frac{8 \pi \gamma_{1}}{\varepsilon_{0} E^{2}}
$$

La décroissance étant exponentielle (5), sa constante de temps est donnée par

$$
T_{\mathrm{D}}=\frac{\gamma_{1}}{4 q_{0}^{2} K_{22}}=\frac{L^{2} \gamma_{1}}{\pi^{2} K_{22}}
$$

Discussion. - Le temps de montée des effets de distorsion, comme celui des effets optiques (Fig. 5b) sont donc proportionnels à l'inverse du carré du champ électrique, pour de faibles valeurs de celui-ci. Ce résultat est à comparer à ceux obtenus dans les effets de champ présentant une transition du type Freedericks où la constante de temps de montée est proportionnelle à $1 /\left(E^{2}-E_{\mathrm{c}}^{2}\right)$ ( $E_{\mathrm{c}}$ est le champ de seuil du phénomène).

La distorsion de la structure précédemment décrite ne présente pas une transition du type Freedericks comme dans le cas plus classique, traité mathématiquement par Berreman [6], où le champ électrique est parallèle à l'axe de l'hélice. D'autre part dans le cas étudié ici, précisons qu'à partir de la Théorie LeslieEricksen $[4,5]$, on montre que, contrairement à l'autre cas, aucun écoulement du liquide (effet backflow) n'accompagne ni ne modifie l'orientation des molécules dans le régime transitoire.

Dans le cas où le champ est parallèle à l'axe de l'hélice, il reste à préciser l'étude mathématique du comportement optique (aspect statique et dynamique) que nous pensons publier prochainement.

\section{Bibliographie}

[1] De Gennes, P. G., Solid State Commun, 6 (1968) 163.

[2] Dreher, R., Solid State Commun. 13 (1973) 1571.

[3] Gharadjedaghi, F., Robert, J., Revue Phys. Appl. 10 (1975) 69 ; Communication présentée à la 5 e Conférence Internationale sur les cristaux liquides : Stockholm (1974).
[4] LesLie, F. M., Quant. J. Mech and Appl. Math. 19 (1966) 357.

[5] Ericksen, J. L., Arch. Rat. Mech. Anal. 23 (1966) 266.

[6] Berreman, D. W., Appl. Phys. Lett. 25 (1974) 12. 